\title{
EXISTENCE AND NONEXISTENCE OF POSITIVE SOLUTIONS FOR SINGULAR SEMILINEAR ELLIPTIC BOUNDARY VALUE PROBLEMS
}

\author{
S. B. Cui(Cui Shangbin, Cui ShangBin) \\ (Department of Mathematics, Lanzhou University, \\ Lanzhou, Gansu 730000, People's Republic of China)
}

\begin{abstract}
In this paper we study existence of positive solutions to singular elliptic boundary value problems involving divergence terms in general domains. By constructing suitable upper and lower solutions and making comparison, we obtain sufficient conditions for existence and nonexistence of solutions. We also study a concrete example to show that the conditions imposed on parameters appearing in the structure conditions of nonlinear terms are optimal and our results can be used to get boundary regularity of solutions.
\end{abstract}

Key words and phrases: Singular elliptic equation, boundary value problem, positive solution, existence, nonexistence.

AMS 1991 classification number: 35J25.

\section{Introduction}

The purpose of this paper is to investigate the existence of solutions to the problem

$$
\left\{\begin{array}{l}
L u+f(x, u, D u)=0, \quad u>0, \quad \text { in } \Omega, \\
u=\varphi, \quad \text { on } \partial \Omega
\end{array}\right.
$$

where $\Omega$ is a bounded smooth domain in $R^{n}, L$ is a second order elliptic partial differential operator on $\Omega, \varphi$ is a nonnegative function defined on $\partial \Omega$, and $f(x, u, \xi)$ is a continuous function defined on $\Omega \times(0, \infty) \times R^{n}$. We are interested in the case where $f(x, u, \xi)$ has singularities on $\partial\left(\Omega \times(0, \infty) \times R^{n}\right)$, i.e., (1.1) is a singular boundary value problem. This kind of problem arises in many applicational fields (c.f.[1-18]).

A typical example of the above problem is as follows:

$$
\left\{\begin{array}{l}
\triangle u+a(x) u^{p}=0, \quad u>0, \quad \text { in } \Omega, \\
u=0, \quad \text { on } \partial \Omega,
\end{array}\right.
$$


where $a(x)$ is locally Hölder continuous and positive in $\Omega$ and $p<0$. This problem was first studied by Crandall et al in 1977 in the case where $\Omega=B=\left\{x \in R^{n}:|x|<1\right\}$ under the assumption that $a(x) \in C^{1}(\bar{B})$ and $\min _{x \in \bar{\Omega}} a(x)>0$. They obtained a unique existence theorem ([1]). Later in 1979 Taliaferro considered the case $n=1$ and $\Omega=(0,1)$, permitting $a(x)$ to be singular at $x=0$ and $x=1$. He proved that a necessary and sufficient condition for the existence of a solution was

$$
\int_{0}^{1} x(1-x) a(x) \mathrm{d} x<\infty([2])
$$

Taliaferro's this result was extended by Usami in 1989 to the case $n \geq 2$ and $\Omega=B([3])$. In fact he considered a more general problem of the form

$$
\left\{\begin{array}{l}
\triangle u+f(x, u)=0, \quad u>0 \quad \text { in } B, \\
u=0, \quad \text { on } \partial B .
\end{array}\right.
$$

His results are as follows. Suppose that $f(x, u)$ is locally Hölder continuous with respect to $(x, u)$ in $B \times(0, \infty)$ and locally Lipchitz continuous with respect to $u$ in $(0, \infty)$ and satisfies

$$
0<f_{*}(|x|, u) \leq f(x, u) \leq f^{*}(|x|, u), \quad \text { for }(x, u) \in B \times(0, \infty),
$$

where $f_{*}$ and $f^{*}$ have similar continuity with $f$ and are strictly monotonically decreasing with respect to $u$ in $(0, \infty)$. Then a sufficient condition for the existence of a solution to the problem (1.4) is that for some $\varepsilon \in(0,1)$ and all $u>0$,

$$
\left\{\begin{array}{lll}
-\int_{1-\varepsilon}^{1}(\log t) f^{*}(t, u) \mathrm{d} t<\infty, & \text { if } & n=2 \\
\int_{1-\varepsilon}^{1}(1-t) f^{*}(t, u) \mathrm{d} t<\infty, & \text { if } & n>2
\end{array}\right.
$$

if inaddition $f^{*}(t, u)$ is convex with respect to $u$ and

$$
\sup _{t \in[0,1), u>0} \frac{f^{*}(t, u)}{f_{*}(t, u)}<\infty
$$

then the condition (1.5) is also necessary. For other related results on the problem (1.4) and the more general problem (1.1) we refer the reader to see [4-9,11,12,14-22].

In this paper, which is a continuation of our previous work $([19,20,23])$, we study the general problem (1.1) in general domains. Our focus attention is paid on how the divergence term $D u$ affects existence of solutions. We prove some existence and nonexistence theorems. These results can be best illustrated by their applications to the following model problem:

$$
\left\{\begin{array}{l}
\triangle u+a(x) u^{p}\left(1+|D u|^{2}\right)^{q / 2}=0, \quad \text { in } \Omega, \\
u=0, \quad \text { on } \partial \Omega,
\end{array}\right.
$$

where $p<1, q<2$ and $a(x)$ is locally Hölder continuous in $\Omega$ and satisfies

$$
C_{1} d(x, \partial \Omega)^{\beta} \leq a(x) \leq C_{2} d(x, \partial \Omega)^{\beta}, \quad \forall x \in \Omega,
$$

with $C_{2} \geq C_{1}>0$ and $\beta \in(-\infty, \infty)$. Here and hereafter we use the notation $d(x, \partial \Omega)$ to denote the distance of $x \in \Omega$ to the boundary of $\Omega$. By our results we have the following conclusions: 
(i) if $p<1-q$ and $\beta>q-2$, then (1.6) has a unique solution;

(ii) if $p \geq 1-q$ and $\beta>-p-1$, then (1.6) has at least one solution if $C_{2}$ is sufficiently small;

(iii) if either $p<1-q$ and $\beta \leq q-2$ or $p \geq 1-q$ and $\beta<-p-1$, then (1.6) has no solution.

From these conclusions we see that in order to get a solution the condition (1.5) can be weakened if $f(x, u, \xi)$ decreases to zero as $|\xi| \rightarrow \infty$, but should be strengthened if $f(x, u, \xi)$ increases to infinitive as $|\xi| \rightarrow \infty$. Besides, these conclusions show that the conditions imposed in our existence and nonexistence theorems on parameters appearing in the structure conditions of $f(x, u, \xi)$ are optimal in certain sense.

The existence theorems established in this paper are refinement and generalizations of those obtained in our previous work $([19,20])$. The nonexistence theorem is thoroughly fresh; it is derived from the comparison theorem proved in our another piece of work [23] where uniqueness of solutions to the problem (1.1) is discussed.

To benefit the reader who wants to get an understanding to the problem (1.2) with $a(x)$ singular on $\partial \Omega$ and $p>1$, we mention the work of Senba et al [13]. They proved that for $\Omega=B$, if $1<p<(N+2) /(N-2)$ and $\beta>\max (-2,-p-1)$ then (1.2) has a solution. We also mention that in the case $p=2$ the problem (1.2) is greatly interested by statists (c.f.[10] and the references cited therein). For general problem (1.1) where $f(x, u, \xi)$ is only singular with respect to $x$ on $\partial \Omega$ and tends to $\infty$ as $u \rightarrow \infty$ at the rate $u^{p}$ with $p>1$, to the best of our knowledge, no results are obtained up to now except in the case $n=1$.

The plan of the following sections are as follows. In Section 2 we enumerate our main results. The other sections are devoted to the proofs of these results.

\section{The Main Results}

Hereafter we always assume that $L$ has form

$$
L=\sum_{i, j=1}^{n} a_{i j}(x) \frac{\partial^{2}}{\partial x_{i} \partial x_{j}}+\sum_{i=1}^{n} b_{i}(x) \frac{\partial}{\partial x_{i}},
$$

where $a_{i j}(x), b_{i}(x) \in C^{\alpha}(\bar{\Omega})$ for some $\alpha \in(0,1), a_{i j}(x)=a_{j i}(x)$, and there exists a constant $\lambda_{0}>0$ such that

$$
\sum_{i, j=1}^{n} a_{i j}(x) \xi_{i} \xi_{j} \geq \lambda_{0}|\xi|^{2}, \quad \forall x \in \Omega, \quad \forall \xi \in R^{n}
$$

We always assume that $\partial \Omega$ is of $C^{1+\alpha}$-class for some $\alpha \in(0,1)$ unless otherwise assumption is specially made. We denote by $\lambda_{1}$ the smallest eigenvalue of the operator $-L: H_{0}^{1}(\Omega) \rightarrow$ $H^{-1}(\Omega)$, and by $\psi(x)$ the corresponding eigenfunction satisfying $\max _{x \in \bar{\Omega}} \psi(x)=1$. It is well-known that $\psi(x) \in C^{2+\alpha}(\Omega) \cap C^{1}(\bar{\Omega})$ and satisfies

$$
\psi(x)>0, \quad \text { for } x \in \Omega ; \quad \psi(x)=0, \quad \frac{\partial \psi}{\partial n}(x)<0, \quad \text { for } x \in \partial \Omega .
$$


Here $n$ represents the outward-pointwising unit normal vector on $\partial \Omega$. From (2.1) we see that there exist constants $C_{1}>0$ and $C_{2}>0$ such that

$$
C_{1} \psi(x) \leq d(x, \partial \Omega) \leq C_{2} \psi(x), \quad \forall x \in \Omega .
$$

In existence theorems to be stated below, a basic assumption is that the function $f(x, u, \xi)$ satisfies the following two conditions:

$\left(D_{1}\right) f(x, u, \xi)$ is locally Hölder continuous in $\Omega \times(0, \infty) \times R^{n}$ and continuously differentiable with respect to the variables $u$ and $\xi$;

$\left(D_{2}\right)$ for any $\Omega_{1} \subset \subset \Omega$ and any $a, b \in(0, \infty)(a<b)$ there exists a corresponding constant $C=C\left(\Omega_{1}, a, b\right)>0$ such that

$$
|f(x, u, \xi)| \leq C\left(1+|\xi|^{2}\right), \quad \forall x \in \overline{\Omega_{1}}, \quad \forall u \in[a, b], \quad \forall \xi \in R^{n} .
$$

These conditions are imposed to guarantee the upper and lower solutions theorem for singular elliptic boundary value problems (see Lemma 3) can be employed.

The existence results are the following three theorems: below:

Theorem 1 Suppose that $f$ satisfies the conditions $\left(D_{1}\right),\left(D_{2}\right)$ and $\left(D_{3}\right),\left(D_{4}\right)$ stated

$\left(D_{3}\right)$ there exist constants $A>0, M>0, k<1$ and $\beta \in(-\infty, \infty)$ such that

$$
f(x, u, \xi) \geq A d(x, \partial \Omega)^{\beta} u^{k}, \quad \forall x \in \Omega, \quad \forall u \in(0, M], \quad \forall|\xi| \leq M ;
$$

$\left(D_{4}\right)$ There exist constants $B_{i}>0(i=1,2, \cdots, m)$ and $\gamma_{i}, p_{i}, q_{i}(i=0,1, \cdots, m)$ satisfying

$$
p_{i}<1, \quad q_{i}<\min \left(2,1-p_{i}\right), \quad \gamma_{i}>q_{i}-2 \quad(i=1,2, \cdots, m)
$$

such that

$$
f(x, u, \xi) \leq \sum_{i=1}^{m} B_{i} d(x, \partial \Omega)^{\gamma_{i}} u^{p_{i}}(1+|\xi|)^{q_{i}}, \quad \forall x \in \Omega, \quad \forall u>0, \quad \forall \xi \in R^{n} .
$$

Then for $\varphi=0$ the problem (1.1) has a classical solution $u$ satisfying the following condition: for any $\mu \geq \max (1, \beta /(1-k))$ and any $\nu \in(0,1) \cap\left(0, \nu^{*}\right]$ there exist corresponding constants $\varepsilon>0$ and $E>0$ such that

$$
\varepsilon d(x, \partial \Omega)^{\mu} \leq u(x) \leq E d(x, \partial \Omega)^{\nu}, \quad \forall x \in \bar{\Omega} .
$$

Here

$$
\nu^{*}=\min _{1 \leq i \leq m}\left(\frac{2+\gamma_{i}-q_{i}}{1-p_{i}-q_{i}}\right) .
$$

Moreover, there exists $\delta>0$ such that for any nonnegative $\varphi \in C^{1+\alpha}(\partial \Omega)$ satisfying $\|\varphi\|_{C^{1+\alpha}(\partial \Omega)} \leq \delta$ the problem (1.1) has a classical solution $u$ satisfying

$$
w(x)+\varepsilon d(x, \partial \Omega)^{\mu} \leq u(x) \leq w(x)+E d(x, \partial \Omega)^{\nu},
$$

where $w$ is the solution of the problem $L w=0$ in $\Omega$ and $\left.w\right|_{\partial \Omega}=\varphi$, and $\mu, \nu, \varepsilon$ and $E$ are constants similar to those appearing in (2.6). below:

Theorem 2 Suppose that $f$ satisfies the conditions $\left(D_{1}\right),\left(D_{2}\right)$ and $\left(D_{3}^{\prime}\right),\left(D_{4}^{\prime}\right)$ stated 
( $\left.D_{3}^{\prime}\right)$ For any given $M>0$, there exist corresponding constants $k<1, \beta \in(-\infty, \infty)$ and $A>0$ such that

$$
f(x, u, \xi) \geq A d(x, \partial \Omega)^{\beta} u^{k}, \quad \forall x \in \Omega, \quad \forall u \in(0, M], \quad \forall|\xi| \leq M .
$$

$\left(D_{4}^{\prime}\right)$ There exist constants $B_{i}>0(i=1,2, \cdots, m)$ and $\gamma_{i}, p_{i}, q_{i}(i=0,1, \cdots, m)$ satisfying

$$
p_{i}<1, \quad q_{i}<\min \left(2,1-p_{i}\right), \quad \gamma_{i}>\max \left(-2, q_{i}-2\right) \quad(i=1,2, \cdots, m)
$$

such that

$$
f(x, u, \xi) \leq \sum_{i=1}^{m} B_{i} d(x, \partial \Omega)^{\gamma_{i}} u^{p_{i}}(1+|\xi|)^{q_{i}}, \quad \forall x \in \Omega, \quad \forall u>0, \quad \forall \xi \in R^{n} .
$$

Then for any nonnegative $\varphi \in C^{1+\alpha}(\partial \Omega)$ the problem (1.1) has a classical solution $u$ satisfying (2.7)

Theorem 3 Suppose that $f$ satisfies the conditons $\left(D_{1}\right),\left(D_{2}\right),\left(D_{3}\right)$ and $\left(D_{4}^{\prime \prime}\right)$ stated below:

$\left(D_{4}^{\prime \prime}\right)$ There exist constants $B_{i}>0(i=1,2, \cdots, m)$ and $\gamma_{i}, p_{i}, q_{i}(i=0,1, \cdots, m)$ satisfying

$$
p_{i}<1, \quad 1-p_{i} \leq q_{i}<2, \quad \gamma_{i}>-p_{i}-1 \quad(i=1,2, \cdots, m)
$$

such that

$$
f(x, u, \xi) \leq \sum_{i=1}^{m} B_{i} d(x, \partial \Omega)^{\gamma_{i}} u^{p_{i}}(1+|\xi|)^{q_{i}}, \quad \forall x \in \Omega, \quad \forall u>0, \quad \forall \xi \in R^{n} .
$$

Then there exists $B_{i}^{*}>0(i=1,2, \cdots, m)$ such that the problem (1.1) with $\varphi=0$ has a classical solution $u$ satisfying (2.6) provided $B_{i} \leq B_{i}^{*}(i=1,2, \cdots, m)$. If in addition all $p_{i}$ 's appearing in the conditon $\left(D_{4}^{\prime \prime}\right)$ are non-positive, then there exist $\delta>0$ and $B_{i}^{*}>0(i=$ $1,2, \cdots, m)$ such that for any nonnegative $\varphi \in C^{1+\alpha}(\partial \Omega)$ satisfying $\|\varphi\|_{C^{1+\alpha}(\partial \Omega)} \leq \delta$ the problem (1.1) has a classical solution $u$ satisfying (2.7) provided $B_{i} \leq B_{i}^{*}(i=1,2, \cdots, m)$.

Remark. A similar result as Theorem 1 above is obtained in [20] (i.e. Theorem 1 therein). As one sees easily, the two conditions $\left(D_{3}\right)$ and $\left(D_{4}\right)$ are respectively weaker than the corresponding conditions required by Theorem 1 of [20]. Thus Theorem 1 above can be regarded as a refinement to that theorem. Theorem 2 and Theorem 3 are entirely new.

The nonexistence result, which is the main contribution of this paper, is as follows.

Theorem 4 Suppose that the function $f$ satisfies the following condtion:

$\left(D_{5}\right)$ there exist constants $A>0$ and $\beta, p$ and $q$ satisfying either

(i) $p<1, q<1-p, \beta \leq q-2$, or

(ii) $p<1, q \geq 1-p, \beta<-p-1$

such that

$$
f(x, u, \xi) \geq A d(x, \partial \Omega)^{\beta} u^{p}(1+|\xi|)^{q}, \quad \forall x \in \Omega, \quad \forall u>0, \quad \forall \xi \in R^{n} .
$$


Then the problem (1.1) has no classical solution for any nonnegative $\varphi \in C(\partial \Omega)$.

Applying these results to the problem (1.6), we obtain

Theorem 5 Suppose that $a(x) \in C^{\alpha}(\Omega)$ for some $\alpha \in(0,1)$ and satisfies the condition (1.7). Suppose furthermore that $p<1$ and $q<2$. Then the following conclusions hold:

(i) If either $q<1-p$ and $\beta \leq q-2$ or $q \geq 1-p$ and $\beta<-p-1$, then the problem (1.6) has no positive classical solution.

(ii) If $q<1-p$ and $\beta>q-2$, then the problem (1.6) has a unique positive classical solution $u(x)$. Moreover, this solution satisfies

$$
\begin{gathered}
C_{1} d(x, \partial \Omega)^{(2+\beta-q) /(1-p-q)} \leq u(x) \leq C_{2} d(x, \partial \Omega)^{(2+\beta-q) /(1-p-q)}, \quad \forall x \in \Omega, \\
\text { if } q-2<\beta<-p-1 ; \\
C_{1} d(x, \partial \Omega) \leq u(x) \leq C_{\nu} d(x, \partial \Omega)^{\nu}, \quad \forall x \in \Omega, \quad \forall \nu \in(0,1) \\
\text { if }-p-1 \leq \beta<-p+1 ; \\
C_{1} d(x, \partial \Omega)^{\beta /(1-p)} \leq u(x) \leq C_{2} d(x, \partial \Omega), \quad \forall x \in \Omega \\
\text { if } \beta \geq-p+1 .
\end{gathered}
$$

(iii) If $q \geq 1-p$ and $\beta>-p-1$, then the problem (1.6) has at least one positive classical solution $u(x)$ when $C_{2}$ appearing in (1.7) is sufficiently small. Moeover, this solution satisfies

$$
C_{1} d(x, \partial \Omega)^{\max (1, \beta /(1-p))} \leq u(x) \leq C_{\nu} d(x, \partial \Omega)^{\nu}, \quad \forall x \in \Omega, \quad \forall \nu \in(0,1) .
$$

In the above $C_{1}, C_{2}$ and $C_{\nu}$ represent positive constants $\left(C_{1}\right.$ and $C_{2}$ are different from those appearing in (1.7)).

The next result is concerned with regularity of solutions to (1.6) obtained by Theorem 5. It is arranged to show that the kinds of estimates like (2.12)-(2.15) can be employed to study boundary regularity of solutions.

Theorem 6 Suppose that the boundary of $\Omega$ is sufficiently smooth. Suppose that $a(x)$ is locally Hölder continuous in $\Omega$ and satisfies the condition (1.7). Suppose furthermore that $n \geq 2, p<1, q<1$ and either $q<1-p, \beta>q-2$ or $q \geq 1-p, \beta>-p-1$. Let $u(x)$ be the solution of the problem (1.6) obtained by Theorem 5. Then we have the following conclusions:

(i) If $q<1-p$ and $\beta>\max \left(q-2, \frac{p q}{1-q}-2\right)$, then $u(x) \in C^{\alpha}(\bar{\Omega})$ for some $\alpha \in(0,1)$.

(ii) If $\beta>-p-1$, then $u(x) \in C^{1+\alpha}(\bar{\Omega})$ for some $\alpha \in(0,1)$.

(iii) If there exists a function $\tilde{d}(x) \in C^{1}(\bar{\Omega})$ satisfying

$$
C_{1} d(x, \partial \Omega) \leq \tilde{d}(x) \leq C_{2} d(x, \partial \Omega), \quad \forall x \in \bar{\Omega}
$$

$\left(C_{1}\right.$ and $C_{2}$ are positive constants) such that $a(x) / \tilde{d}(x)^{\beta}$ is uniformly Hölder continuous in $\Omega$, and in addition, $\beta>-p$, then $u(x) \in C^{2+\alpha}(\bar{\Omega})$ for some $\alpha \in(0,1)$.

In the last theorem we have used the notation $C^{\alpha}(\bar{\Omega})$ to denote the set of functions on $\Omega$ which are uniformly Hölder continuous in $\Omega$ of order $\alpha$, i.e., $u(x) \in C^{\alpha}(\bar{\Omega})$ if and only if $u(x)$ can be extended to $\bar{\Omega}$ such that the extension is Hölder continuous on $\bar{\Omega}$ of order $\alpha$. The notation $C^{1+\alpha}(\bar{\Omega}), C^{2+\alpha}(\bar{\Omega})$ has similar meaning. 
Remark. We note that if $q-2<\beta<-p-1$ then by (2.12) we have $|D u| \rightarrow \infty$ as $x \rightarrow \partial \Omega$. Therefore, $u(x) \notin C^{1}(\bar{\Omega})$ in this case. We also note that if $-p-1<\beta<-p$ then $u \in C^{1}(\bar{\Omega})$ by the conclusion (ii) and $a(x) u^{p} \rightarrow \infty$ as $x \rightarrow \partial \Omega$ by (2.13) and (2.15), which implies that $\Delta u \rightarrow-\infty$ as $x \rightarrow \partial \Omega$. Therefore, $u \notin C^{2}(\bar{\Omega})$ in this case.

\section{The Proofs of Theorem 1, 2 and 3}

To prove these theorems we first establish three lemmas.

Lemma 1 Suppose that the function $f(x, u, \xi)$ satisfies the condition $\left(D_{3}\right)$. Then for any $\mu \geq \max (1, \beta /(1-k))$ there exists a corresponding $\varepsilon^{*}>0$ such that for every $\varepsilon \in\left(0, \varepsilon^{*}\right], \quad \underline{u}(x)=\varepsilon \psi(x)^{\mu}$ is a lower solution of the problem (1.1) with $\varphi=0$.

Proof. First we note that

$$
\begin{aligned}
D \underline{u}(x) & =\varepsilon \mu \psi(x)^{\mu-1} D \psi(x), \\
L \underline{u}(x) & =\varepsilon \mu \psi(x)^{\mu-1} L \psi(x)+\varepsilon \mu(\mu-1) \psi(x)^{\mu-2} \sum_{i, j=1}^{n} a_{i j}(x) \frac{\partial \psi}{\partial x_{i}}(x) \frac{\partial \psi}{\partial x_{j}}(x) \\
& \geq-\varepsilon \lambda_{1} \mu \psi(x)^{\mu} .
\end{aligned}
$$

Now let $\varepsilon>0$ be so small that both it and $\varepsilon \mu \max _{x \in \bar{\Omega}}|D \psi(x)|$ are not greater than the number $M$ appearing in (2.4). Then we have

$$
\begin{aligned}
L \underline{u}+f(x, \underline{u}, D \underline{u}) & \geq-\varepsilon \lambda_{1} \mu \psi(x)^{\mu}+A \varepsilon^{k} d(x, \partial \Omega)^{\beta} \psi(x)^{\mu k} \\
& \geq A^{\prime} \varepsilon^{k} \psi(x)^{\beta+\mu k}-\varepsilon \lambda_{1} \mu \psi(x)^{\mu} \\
& \geq \varepsilon^{k} \psi(x)^{\beta+\mu k}\left[A^{\prime}-\lambda_{1} \mu \varepsilon^{1-k} \psi(x)^{\mu(1-k)-\beta}\right],
\end{aligned}
$$

where $A^{\prime}=A C_{1}^{\beta}$ (if $\beta \geq 0$ ) or $A C_{2}^{\beta}$ (if $\beta<0$ ). Since $\mu(1-k)-\beta \geq 0$ and $0 \leq \psi(x) \leq 1$, we see that $\psi(x)^{\mu(1-k)-\beta} \leq 1$. Thus

$$
L \underline{u}+f(x, \underline{u}, D \underline{u}) \geq \varepsilon^{p} \psi(x)^{\beta+\mu k}\left[A^{\prime}-\lambda_{1} \mu \varepsilon^{1-k}\right]
$$

which combined with the fact $k<1$ implies that for sufficiently small $\varepsilon>0$,

$$
L \underline{u}+f(x, \underline{u}, D \underline{u}) \geq 0, \quad \text { in } \Omega,
$$

namely, $\underline{u}(x)=\varepsilon \psi(x)^{\mu}$ is a lower solution of the problem (1.1) with $\varphi=0$. Q. E. D.

Lemma 2 Suppose that the function $f(x, u, \xi)$ satisfies the condition $\left(D_{4}\right)$. Then for any $\nu \in(0,1) \cap\left(0, \nu^{*}\right]$, where $\nu^{*}$ is as in Theorem 1 , there exist a corresponding constant $E_{\nu}^{*}>0$ such that for every $E \geq E_{\nu}^{*}, \bar{u}(x)=E \psi(x)^{\nu}$ is an upper solution of the problem (1.1) with $\varphi=0$.

Proof. Without loss of generality we assume that

$$
q_{i} \leq 0\left(i=1,2, \cdots, i_{0}\right) \quad \text { and } \quad q_{i}>0\left(i=i_{0}+1, \cdots, m\right)
$$


where $0 \leq i_{0} \leq m$. Then for arbitrary $E \geq 1$ we have

$$
\begin{aligned}
L \bar{u}+f(x, \bar{u}, D \bar{u}) \leq & -\lambda_{1} \nu E \psi(x)^{\nu}-\nu(1-\nu) E \psi(x)^{\nu-2} \sum_{i, j=1}^{n} a_{i j}(x) \frac{\partial \psi}{\partial x_{i}}(x) \frac{\partial \psi}{\partial x_{j}}(x) \\
& +\sum_{i=1}^{i_{0}} B_{i} E^{p_{i}} d(x, \partial \Omega)^{\gamma_{i}} \psi(x)^{\nu p_{i}}\left(1+E \nu \psi(x)^{\nu-1}|D \psi(x)|\right)^{q_{i}} \\
& +\sum_{i=i_{0}+1}^{m} B_{i} E^{p_{i}} d(x, \partial \Omega)^{\gamma_{i}} \psi(x)^{\nu p_{i}}\left(1+E \nu \psi(x)^{\nu-1}|D \psi(x)|\right)^{q_{i}} \\
\leq & -\lambda_{1} \nu E \psi(x)^{\nu}-\lambda_{0} \nu(1-\nu) E \psi(x)^{\nu-2}|D \psi(x)|^{2} \\
& +\sum_{i=1}^{i_{0}} B_{i}^{\prime} E^{p_{i}} \psi(x)^{\gamma_{i}+\nu p_{i}-(1-\nu) q_{i}}\left(\psi(x)^{1-\nu}+\nu|D \psi(x)|\right)^{q_{i}} \\
& +\sum_{i=i_{0}+1}^{m} B_{i}^{\prime} E^{p_{i}+q_{i}} \psi(x)^{\gamma_{i}+\nu p_{i}-(1-\nu) q_{i}}\left(\psi(x)^{1-\nu}+\nu|D \psi(x)|\right)^{q_{i}} \\
\leq & E \psi(x)^{\nu-2}\left\{-\nu\left(\lambda_{1} \psi(x)^{2}+\lambda_{0}(1-\nu)|D \psi(x)|^{2}\right)\right. \\
& +\sum_{i=1}^{i_{0}} B_{i}^{\prime} E^{-\left(1-p_{i}\right)} \psi(x)^{2+\gamma_{i}-q_{i}-\left(1-p_{1}-q_{i}\right) \nu}\left(\psi(x)^{1-\nu}+\nu|D \psi(x)|\right)^{q_{i}} \\
& \left.+\sum_{i=i_{0}+1}^{m} B_{i}^{\prime} E^{-\left(1-p_{i}-q_{i}\right)} \psi(x)^{2+\gamma_{i}-q_{i}-\left(1-p_{i}-q_{i}\right) \nu}\left(\psi(x)^{1-\nu}+\nu|D \psi(x)|\right)^{q_{i}}\right\} .
\end{aligned}
$$

Let

$$
\begin{gathered}
M_{0}=\min _{x \in \bar{\Omega}}\left(\lambda_{1} \psi(x)^{2}+\lambda_{0}(1-\nu)|D \psi(x)|^{2}\right), M_{1}=\min _{x \in \bar{\Omega}}\left(\psi(x)^{1-\nu}+\nu|D \psi(x)|\right), \\
M_{2}=\max _{x \in \bar{\Omega}}\left(\psi(x)^{1-\nu}+\nu|D \psi(x)|\right) .
\end{gathered}
$$

It is obvious that $M_{0}>0, M_{1}>0$ and $M_{2}<\infty$. Therefore, since all the exponents of $\psi(x)$ in the big brackets are nonnegative, we have

$$
L \bar{u}+f(x, \bar{u}, D \bar{u}) \leq E \psi(x)^{\nu-2}\left\{-\nu M_{0}+\sum_{i=1}^{i_{0}} B_{i}^{\prime} M_{1}^{q_{i}} E^{-\left(1-p_{i}\right)}+\sum_{i=i_{0}+1}^{m} B_{i}^{\prime} M_{2}^{q_{i}} E^{-\left(1-p_{i}-q_{i}\right)}\right\} .
$$

By the hypothesis, all the exponents of $E$ in the last pair of brackets are negative. Hence, for sufficiently large $E$ we have

$$
L \bar{u}+f(x, \bar{u}, D \bar{u}) \leq 0, \quad \text { in } \Omega ;
$$

namely, $\bar{u}(x)=E \psi(x)^{\nu}$ is an upper solution for sufficiently large E. Q. E. D.

Lemma 3 Suppose that the function $f$ satisfies the conditions $\left(D_{1}\right)$ and $\left(D_{2}\right)$. Suppose furthermore that the problem (1.1) has a pair of upper and lower solutions $\bar{u}(x)$ and $\underline{u}(x)$ satisfying the conditions

(1) $\bar{u}(x), \underline{u}(x) \in C^{2}(\Omega) \cap C(\bar{\Omega})$;

(2) $0<\underline{u}(x) \leq \bar{u}(x), \quad \forall x \in \Omega$;

(3) $\bar{u}(x)=\underline{u}(x)=\varphi(x), \quad \forall x \in \partial \Omega$.

Then this problem has a solution $u(x)$ belonging to $C^{2+\alpha}(\Omega) \cap C(\bar{\Omega})$ and satisfying

$$
\underline{u}(x) \leq u(x) \leq \bar{u}(x), \quad \forall x \in \bar{\Omega} .
$$

This lemma is due to Li Jian-zhang. It can be proved by utilizing the domain approximation method $([3,19,20,24])$. Since it has been actually proved in [20], we omit its proof here. 
Proof of Theorem 1. The first conclusion of Theorem 1 follows directly from Lemma 1, 2 and 3. To prove the second conclusion, we denote $g(x, v, \eta)=f(x, v+w(x), \eta+D w(x))$, where $w$ is as in Theorem 1 . Let $\delta>0$ be sufficiently small such that $\max _{x \in \bar{\Omega}}\{w(x),|D w(x)|\} \leq$ $\min \{1 / 2, M / 2\}$ when $\|\varphi\|_{C^{1+\alpha}(\partial \Omega)} \leq \delta$, where $M$ is the constant involved in the condition $\left(D_{3}\right)$. Now let $\varphi$ satisfies this condition. Then since $w(x) \geq \min _{x \in \partial \Omega} \varphi(x) \geq 0$ by maximum principle, we have

$$
\begin{aligned}
& (v+w(x))^{k} \geq\left\{\begin{array}{l}
v^{k}, \quad \text { if } k \geq 0, \\
M^{k}, \quad \text { if } k<0,
\end{array} \quad \forall x \in \Omega, \quad \forall 0<v \leq \frac{M}{2} ;\right. \\
& (v+w(x))^{p_{i}} \leq\left\{\begin{array}{l}
v^{p_{i}}, \quad \text { if } p_{i} \leq 0, \\
v^{p_{i}}+\left(\frac{M}{2}\right)^{p_{i}}, \text { if } 0<p_{i}<1,
\end{array} \quad \forall x \in \Omega, \quad \forall v>0 ;\right. \\
& (1+|\eta+D w(x)|)^{q_{i}} \leq\left\{\begin{array}{ll}
\left(\frac{3}{2}\right)^{q_{i}}(1+|\eta|)^{q_{i}}, & \text { if } 0 \leq q_{i}<2, \\
\left(\frac{1}{2}\right)^{q_{i}}(1+|\eta|)^{q_{i}}, & \text { if } q_{i}<, .
\end{array} \quad \forall x \in \Omega, \quad \forall \eta \in R^{n} .\right.
\end{aligned}
$$

Applying these inequalities, we get from (2.4) and (2.5) respectively

$$
\begin{gathered}
g(x, v, \eta)=f(x, v+w(x), \eta+D w(x)) \geq A d(x, \partial \Omega)^{\beta}(v+w(x))^{k} \\
\geq A^{\prime} d(x, \partial \Omega)^{\beta} v^{k^{\prime}}, \quad \forall x \in \Omega, \quad \forall v \in\left(0, \frac{M}{2}\right], \quad \forall|\eta| \leq \frac{M}{2}, \\
\left.g(x, v, \eta) \leq \sum_{i=1}^{m} B_{i} d(x, \partial \Omega)^{\gamma_{i}}(v+w(x))\right)^{p_{i}}(1+|\eta+D w(x)|)^{q_{i}} \\
\leq \sum_{i=1}^{m^{\prime}} B_{i}^{\prime} d(x, \partial \Omega)^{\gamma_{i}^{\prime}} v^{p_{i}^{\prime}}(1+|\eta|)^{q_{i}^{\prime}}, \quad \forall x \in \Omega, \quad \forall v>0, \quad \forall \eta \in R^{n},
\end{gathered}
$$

where $k^{\prime}=k$ for $k \geq 0$ and 0 for $k<0, m^{\prime}$ is a positive integer, $\gamma_{i}^{\prime}, p_{i}^{\prime}, q_{i}^{\prime}\left(i=1,2, \cdots, m^{\prime}\right)$ are constants satisfying some inequalities similar to those satisfied by $\gamma_{i}, p_{i}, q_{i}(i=1,2, \cdots, m)$ in the condition $\left(D_{4}\right)$, and $A^{\prime}$ and $B_{i}^{\prime}\left(i=1,2, \cdots, m^{\prime}\right)$ are positive constants. Therefore, $g(x, v, \eta)$ satisfies the condition $\left(D_{3}\right)$ and $\left(D_{4}\right)$. Consequently, by Lemma 1 and 2 we see that there exist $\mu \geq 1$ and $\nu \in(0,1)$ such that for sufficiently small $\varepsilon>0$ and sufficiently large $E>0, \underline{v}(x)=\varepsilon \psi(x)^{\mu}$ and $\bar{v}(x)=E \psi(x)^{\nu}$ are respectively lower and upper solutions of the problem

$$
\left\{\begin{array}{l}
L v+g(x, v, D v)=0, v>0, \text { in } \Omega \\
v=0, \text { on } \partial \Omega .
\end{array}\right.
$$

Hence, by Lemma 3, (3.2) has a classical solution $v$ satisfying $\varepsilon \psi(x)^{\mu} \leq v(x) \leq E \psi(x)^{\nu}$. Evidently, $u=v+w$ is then a solution to the problem (1.1) satisfying (2.7). This finishes the proof of Theorem 1. Q. E. D.

Proof of Theorem 2. Let the notation be as above. Let $N=\max _{x \in \bar{\Omega}}\{w(x),|D w(x)|\}$. Then we have

$$
\begin{aligned}
& (v+w(x))^{p_{i}} \leq\left\{\begin{array}{l}
v^{p_{i}}, \quad \text { if } p_{i} \leq 0, \\
v^{p_{i}}+N^{p_{i}}, \text { if } 0<p_{i}<1,
\end{array} \quad \forall x \in \Omega, \quad \forall v>0\right. \\
& (1+|\eta+D w(x)|)^{q_{i}} \leq\left\{\begin{array}{l}
(1+N)^{q_{i}}(1+|\eta|)^{q_{i}}, \text { if } 0 \leq q_{i}<2, \\
1, \quad \text { if } q_{i}<0,
\end{array} \forall x \in \Omega, \quad \forall \eta \in R^{n} .\right.
\end{aligned}
$$


By applying these inequalities one may verify that the condition $\left(D_{4}^{\prime}\right)$ implies that $g(x, v, \eta)$ satisfies the condition $\left(D_{4}\right)$. Therefore, by Lemma 2 we see that there exists $\nu \in(0,1)$ such that for sufficiently large $E, \bar{v}(x)=E \psi(x)^{\nu}$ is an upper solution of the problem (3.2). Next we take $M=N+1$ and apply the condition $\left(D_{3}^{\prime}\right)$. Then we get

$$
g(x, v, \eta) \geq\left\{\begin{array}{l}
A d(x, \partial \Omega)^{\beta} v^{k}, \quad \text { if } 0 \leq k<1 \text { and } v \in(0,1), \quad|\eta| \leq 1, \\
A d(x, \partial \Omega)^{\beta} M^{k}, \quad \text { if } k<0 \text { and } v \in(0,1), \quad|\eta| \leq 1 .
\end{array}\right.
$$

This shows that $g(x, v, \eta)$ satisfies the condition $\left(D_{3}\right)$ with $k=k$ for $0 \leq k<1$ and 0 for $k<0$. Therefore, by Lemma 1 we see that there exists $\mu \geq 1$ such that for sufficiently small $\varepsilon>0, \underline{v}(x)=\varepsilon \psi(x)^{\mu}$ is a lower solution of the problem (3.2). Now by an similar argument as we have made in the proof of Theorem 1 we get the conclusion of Theorem 2. Q. E. D.

Proof of Theorem 3. Let $\nu_{i}=\left(q_{i}-2-\gamma_{i}\right) /\left(p_{i}+q_{i}-1\right)$ if $q_{i}>1-p_{i}$ and $\nu_{i}=-\infty$ if $q_{i}=1-p_{i}(i=1,2, \cdots, m)$. Let $\nu_{*}=\max _{1 \leq i \leq m} \nu_{i}$. Choose a number $\nu \in(0,1)$ such that $\nu \geq \nu_{*}$. Since obviously $\nu_{*}<1$, such $\nu$ exists. Let $\bar{u}(x)=\psi(x)^{\nu}$, and let $M_{0}, M_{2}$ be as in (3.1). Then we have

$$
\begin{aligned}
L \bar{u}+f(x, \bar{u}, D \bar{u}) \leq & -\lambda_{1} \nu \psi^{\nu}-\nu(1-\nu) \lambda_{0} \psi^{\nu-2}|D \psi|^{2} \\
& +\sum_{i=1}^{m} B_{i}^{\prime} \psi^{\gamma_{i}+\nu p_{i}}\left(1+\nu \psi^{\nu-1}|D \psi|\right)_{i}^{q} \\
\leq & -\nu M_{0} \psi^{\nu-2}+\sum_{i=1}^{m} B_{i}^{\prime} M_{2}^{q_{i}} \psi^{\gamma_{i}+\nu p_{i}-(1-\nu) q_{i}} \\
= & \psi^{\nu-2}\left\{-\nu M_{0}+\sum_{i=1}^{m} B_{i}^{\prime} M_{2}^{q_{i}} \psi^{2+\gamma_{i}-q_{i}+\nu\left(p_{i}+q_{i}-1\right)}\right\}
\end{aligned}
$$

where $B_{i}^{\prime}=B_{i} C_{2}^{\gamma_{i}}$ (if $\gamma_{i} \geq 0$ ) or $B_{i} C_{1}^{\gamma_{i}}$ (if $\gamma_{i}<0$ ). Since $2+\gamma_{i}-q_{i}+\nu\left(p_{i}+q_{i}-1\right) \geq$ $2+\gamma_{i}-q_{i}+\nu_{*}\left(p_{i}+q_{i}-1\right) \geq 0$, we see that $\psi^{2+\gamma_{i}-q_{i}+\nu\left(p_{i}+q_{i}-1\right)} \leq 1$. Therefore, $\bar{u}(x)=\psi(x)^{\nu}$ is an upper solution of the problem (1.1) with $\varphi=0$ when $B_{i}^{\prime} \leq \nu M_{0} / m M_{2}^{q_{i}}$. Now by making application of Lemma 1 and 3 we get the first conclusion of Theorem 3 . To prove the second conclusion, let $w(x)$ and $g(x, v, \eta)$ be as in the proof of Theorem 1 and suppose that $\max _{x \in \bar{\Omega}}|D w(x)| \leq \min \{1, M / 2\}$, where $M$ is the number appearing in the condition $\left(D_{3}\right)$. Since $p_{i} \leq 0(i=1,2, \cdots, m)$ and $q_{i} \geq 1-p_{i}>0(i=1,2, \cdots, m)$, we have

$$
\begin{aligned}
g(x, v, \eta) & \leq \sum_{i=1}^{m} B_{i} d(x, \partial \Omega)^{\gamma_{i}}(v+w(x))^{p_{i}}(1+|\eta+D w(x)|)^{q_{i}} \\
& \leq \sum_{i=1}^{m} B_{i} d(x, \partial \Omega)^{\gamma_{i}} v^{p_{i}}(2+|\eta|)^{q_{i}}, \quad \forall x \in \Omega, \quad \forall v>0, \quad \forall \eta \in R^{n} .
\end{aligned}
$$

Thus by a similar argument as we have made above shows that there exist $B_{i}^{*}>0(i=$ $1,2, \cdots, m)$ such that when $B_{i} \leq B_{i}^{*}(i=1,2, \cdots, m), \bar{v}(x)=\psi(x)^{\nu}$ is an upper solution of the problem (3.2), where $\nu$ is as above. Besides, from the proof of the second conclusion of Theorem 1 we see that there exists $\mu \in(0,1)$ such that $\underline{v}(x)=\varepsilon \psi(x)^{\mu}$ is a lower solution of the problem (3.2). Therefore, by repeating the argument we have made in the proof of the second conclusion of Theorem 1 we get the second conclusion of Theorem 3. Q.E.D.

\section{The Proof of Theorem 4}

The proof of Theorem 4 is based on comparison between upper and lower solutions of the following problem:

$$
\left\{\begin{array}{l}
L u+h(x, u, D u)=0, \quad u>0, \quad \text { in } \Omega \\
u=\varphi \geq 0, \text { on } \partial \Omega
\end{array}\right.
$$


where

$$
h(x, u, \xi)=A \psi(x)^{\beta} u^{p}(1+|\xi|)^{q}, \quad A>0, p<1, q<1-p, \beta \in(-\infty, \infty) .
$$

$\psi(x)$ is as before. For this problem we have

Lemma 4 Suppose that the above conditions for $p$ and $q$ are satisfied. Then we have the following comparison: if two functions $\bar{u}(x), \underline{u}(x) \in C^{2}(\Omega) \cap C(\bar{\Omega})$ are respectively upper and lower solutions of the problem (4.1)-(4.2), then $\bar{u}(x) \geq \underline{u}(x)$ for all $x \in \bar{\Omega}$.

Proof. Choose a positive number $\mu$ such that

$$
\begin{cases}p<\mu<1, & \text { if } q \leq 0: \\ p /(1-q)<\mu<1, & \text { if } 0<q<1 \\ \mu<1, & \text { if } q=1 \\ \mu<\min (1,-p /(q-1)), & \text { if } q>1 .\end{cases}
$$

Then $g(s)=s^{\mu}$ is a test function (c.f [23] for the definition of this concept). One may easily verify that the function $h(x, u, \xi)=A \psi(x)^{\beta} u^{p}(1+|\xi|)^{q}$ satisfies the condition of [23, Theorem 2.1] with $g(s)$ taken in this form (c.f. the proof of [23, Theorem 4.1]). Therefore, by applying [23, Theorem 2.1] we get the conclusion of the above lemma. Q. E. D.

Proof of Theorem 4. By virtue of (2.2), the inequality (2.11) can be rewritten as

$$
f(x, u, \xi) \geq A^{\prime} \psi(x)^{\beta} u^{p}(1+|\xi|)^{q}, \quad \forall x \in \Omega, \quad \forall u>0, \quad \forall \xi \in R^{n},
$$

where $A^{\prime}>0$. We first assume that $p, q$ and $\beta$ satisfy the condition $(i)$, namely, $p<1$, $q<1-p$ and $\beta \leq q-2$. Let us denote

$$
\begin{gathered}
N_{0}=\max _{x \in \bar{\Omega}}\left(\lambda_{1} \psi(x)^{2}+\lambda_{0}|D \psi(x)|^{2}\right), \quad N_{1}=\min _{x \in \bar{\Omega}}(\psi(x)+|D \psi(x)|), \quad N_{2}=\max _{x \in \bar{\Omega}}(1+|D \psi(x)|), \\
h(x, u, \xi)=A^{\prime} \psi(x)^{\beta} u^{p}(1+|\xi|)^{q},
\end{gathered}
$$

where $\lambda_{0}$ and $\lambda_{1}$ are as in the previous section. It is evident that $N_{0}<\infty, N_{1}>0$ and $N_{2}<\infty$. We now make the discussion according to the four different cases $p \geq 0, q \geq 0$; $p \geq 0, q<0 ; \quad p<0, q \geq 0$ and $p<0, q<0$ separately.

Case 1: $p \geq 0$ and $q \geq 0$. In this case we choose a number $\varepsilon \in(0,1)$ so small that $C^{\prime} N_{1}^{q}-\varepsilon^{1-p-q} N_{0} \geq 0$. Since $p+q<1$, this is feasible. With $\varepsilon$ taken in this way, for each $\nu \in(0,1)$ we denote $u_{\nu}(x)=\varepsilon \nu^{-1 /(1-p)} \psi(x)^{\nu}$. Then by $(4.2)$ we have

$$
\begin{aligned}
L u_{\nu}+h\left(x, u_{\nu}, D u_{\nu}\right)= & -\lambda_{1} \varepsilon \nu^{-1 /(1-p)+1} \psi(x)^{\nu}-\lambda_{0} \varepsilon \nu^{-1 /(1-p)+1}(1-\nu) \psi(x)^{\nu-2}|D \psi(x)|^{2} \\
& +A^{\prime} \varepsilon^{p} \nu^{-p /(1-p)} \psi(x)^{\beta+\nu p}\left(1+\varepsilon \nu^{-1 /(1-p)+1} \psi(x)^{\nu-1}|D \psi(x)|\right)^{q} \\
\geq & -\varepsilon \nu^{-p /(1-p)} \psi(x)^{\nu-2}\left(\lambda_{1} \psi(x)^{2}+\lambda_{0}|D \psi(x)|^{2}\right) \\
& +A^{\prime} \varepsilon^{p+q} \nu^{-p /(1-p)} \psi(x)^{\beta+\nu p-(1-\nu) q}\left(\varepsilon^{-1} \psi(x)^{1-\nu}+|D \psi(x)|\right)^{q} \\
\geq & -\varepsilon N_{0} \nu^{-p /(1-p)} \psi(x)^{\nu-2}+A^{\prime} N_{1}^{q} \varepsilon^{p+q} \nu^{-p /(1-p)} \psi(x)^{\beta+\nu p-(1-\nu) q} \\
= & \varepsilon^{p+q} \nu^{-p /(1-p)} \psi(x)^{\beta+\nu p-(1-\nu) q}\left\{A^{\prime} N_{1}^{q}\right. \\
& \left.-\varepsilon^{1-p-q} N_{0} \psi(x)^{\nu(1-p-q)+q-\beta-2}\right\} \\
\geq & \varepsilon^{p+q} \nu^{-p /(1-p)} \psi(x)^{\beta+\nu p-(1-\nu) q}\left\{A^{\prime} N_{1}^{q}-\varepsilon^{1-p-q} N_{0}\right\} .
\end{aligned}
$$


The last inequality is due to the fact that $1-p-q>0$ and $q-\beta-2 \geq 0$. By the choice of the number $\varepsilon$, we see that

$$
L u_{\nu}+h\left(x, u_{\nu}, D u_{\nu}\right) \geq 0, \quad \text { in } \Omega,
$$

which implies that $u_{\nu}(x)$ is a lower solution of the problem (4.1) with $h(x, u, \xi)$ given by (4.4). On the other hand, it is evident that if $u(x)$ is a classical solution of the problem (1.1), then it is an upper solution of the problem (4.1). Therefore, by Lemma 3 we conclude that $u(x) \geq u_{\nu}(x)$ in $\Omega$, namely,

$$
u(x) \geq \varepsilon \nu^{-1 /(1-p)} \psi(x)^{\nu}, \quad \forall x \in \Omega .
$$

Since this inequality holds for all $\nu \in(0,1)$, by letting $\nu \rightarrow 0$ we get $u(x)=\infty, \forall x \in \Omega$. This is an absurdity. Hence (1.1) and (1.2) can not have a classical solution.

Case 2: $p \geq 0$ and $q<0$. In this case we choose a number $\varepsilon \in(0,1)$ so small that $C^{\prime} N_{2}^{q}-\varepsilon^{1-p} N_{0} \geq 0$, and then for each $\nu \in(0,1)$ let $u_{\nu}(x)=\varepsilon \nu^{-(1-q) /(1-p-q)} \psi(x)^{\nu}$. Then we have

$$
\begin{aligned}
L u_{\nu}+h\left(x, u_{\nu}, D u_{\nu}\right)= & -\lambda_{1} \varepsilon \nu^{-(1-q) /(1-p-q)+1} \psi(x)^{\nu} \\
& -\lambda_{0} \varepsilon \nu^{-(1-q) /(1-p-q)+1}(1-\nu) \psi(x)^{\nu-2}|D \psi(x)|^{2} \\
& +A^{\prime} \varepsilon^{p} \nu^{-p(1-q) /(1-p-q)} \psi(x)^{\beta+\nu p} \\
& \cdot\left(1+\varepsilon \nu^{-(1-q) /(1-p-q)+1} \psi(x)^{\nu-1}|D \psi(x)|\right)^{q} \\
\geq & -\varepsilon \nu^{-p /(1-p-q)} \psi(x)^{\nu-2}\left(\lambda_{1} \psi(x)^{2}+\lambda_{0}|D \psi(x)|^{2}\right) \\
& +A^{\prime} \varepsilon^{p} \nu^{-p(1-q) /(1-p-q)-p q /(1-p-q)} \psi(x)^{\beta+\nu p-(1-\nu) q} \\
& \cdot\left(\nu^{p /(1-p-q)} \psi(x)^{1-\nu}+|D \psi(x)|\right)^{q} \\
\geq & \varepsilon^{p} \nu^{-p /(1-p-q)} \psi(x)^{\beta+\nu p-(1-\nu) q}\left\{A^{\prime} N_{2}-\varepsilon^{1-p} N_{0}\right\} .
\end{aligned}
$$

By the choice of $\varepsilon$, we see that

$$
L u_{\nu}+h\left(x, u_{\nu}, D u_{\nu}\right) \geq 0, \quad \text { in } \Omega .
$$

From this, by a similar deduction as in the case 1 we get the conclusion that the problem (1.1) can not have a classical solution.

Case 3: $p<0$ and $q \geq 0$. In this case we still let $u_{\nu}(x)=\varepsilon \nu^{-(1-q) /(1-p-q)} \psi(x)^{\nu}$, where $\nu$ is as before, but $\varepsilon$ is determined by the conditions $A^{\prime} N_{1}^{q}-\varepsilon^{1-p-q} N_{0} \geq 0$ and $0<\varepsilon<1$. Then we have

$$
\begin{aligned}
L u_{\nu}+h\left(x, u_{\nu}, D u_{\nu}\right)= & -\varepsilon \nu^{-p /(1-p-q)} \psi(x)^{\nu-2}\left(\lambda_{1} \psi(x)^{2}+\lambda_{0}|D \psi(x)|^{2}\right) \\
& +A^{\prime} \varepsilon^{p} \nu^{-p(1-q) /(1-p-q)} \psi(x)^{\beta+\nu p} \\
& \cdot\left(1+\varepsilon \nu^{-p /(1-p-q)} \psi(x)^{\nu-1}|D \psi(x)|\right)^{q} \\
\geq & -\varepsilon \nu^{-p /(1-p-q)} \psi(x)^{\nu-2}\left(\lambda_{1} \psi(x)^{2}+\lambda_{0}|D \psi(x)|^{2}\right) \\
& +A^{\prime} \varepsilon^{p+q} \nu^{-p /(1-p-q)} \psi(x)^{\beta+\nu p-(1-\nu) q} \\
& \cdot\left(\varepsilon^{-1} \nu^{p /(1-p-q)} \psi(x)^{1-\nu}+|D \psi(x)|\right)^{q} \\
\geq & \varepsilon^{p+q} \nu^{-p /(1-p-q)} \psi(x)^{\beta+\nu p-(1-\nu) q}\left\{A^{\prime} N_{1}^{q}-\varepsilon^{1-p-q} N_{0}\right\} \\
\geq & 0, \quad \text { in } \Omega .
\end{aligned}
$$


Consequently, the problem (1.1) can not have a classical solution.

Case 4: $p<0$ and $q<0$. In this case we let $u_{\nu}(x)$ as in the case 1 , i.e. $u_{\nu}(x)=$ $\varepsilon \nu^{-1 /(1-p)} \psi(x)^{\nu}$, but with $\varepsilon$ determined by the conditions $A^{\prime} N_{2}^{q}-\varepsilon^{1-p} N_{0} \geq 0$ and $0<\varepsilon<1$. Then we have

$$
\begin{aligned}
L u_{\nu}+h\left(x, u_{\nu}, D u_{\nu}\right)= & -\varepsilon \nu^{-p /(1-p)} \psi(x)^{\nu-2}\left(\lambda_{1} \psi(x)^{2}+\lambda_{0}|D \psi(x)|^{2}\right) \\
& +A^{\prime} \varepsilon^{p} \nu^{-p /(1-p)} \psi(x)^{\beta+\nu p}\left(1+\varepsilon \nu^{-p /(1-p)} \psi(x)^{\nu-1}|D \psi(x)|\right)^{q} \\
\geq & -\varepsilon \nu^{-p /(1-p)} \psi(x)^{\nu-2}\left(\lambda_{1} \psi(x)^{2}+\lambda_{0}|D \psi(x)|^{2}\right) \\
& +A^{\prime} \varepsilon^{p} \nu^{-p /(1-p)} \psi(x)^{\beta+\nu p-(1-\nu) q}\left(\psi(x)^{1-\nu}+|D \psi(x)|\right)^{q} \\
\geq & \varepsilon^{p} \nu^{-p /(1-p)} \psi(x)^{\beta+\nu p-(1-\nu) q}\left\{A^{\prime} N_{2}^{q}-\varepsilon^{1-p} N_{0}\right\} \\
\geq & 0, \quad \text { in } \Omega .
\end{aligned}
$$

Consequently, the problem (1.1) can not have a classical solution.

Next we assume that $p, q$ and $\beta$ satisfy the condition (ii), namely, $p<1, q \geq 1-p$ and $\beta<-p-1$. From $\beta<-p-1$ we see that $\beta+2<1-p$. Thus a number $q_{1}$ can be chosen such that $\beta+2 \leq q_{1}<1-p$. We denote

$$
h(x, u, \xi)=A^{\prime} \psi(x)^{\beta} u^{p}(1+|\xi|)^{q_{1}} .
$$

Suppose that the problem (1.1) has a classical solution $u(x)$. Then

$$
\begin{aligned}
L u+h(x, u, D u) & =-f(x, u, D u)+A^{\prime} \psi(x)^{\beta} u^{p}(1+|D u|)^{q_{1}} \\
& \leq-A^{\prime} \psi(x)^{\beta} u^{p}(1+|D u|)^{q}+A^{\prime} \psi(x)^{\beta} u^{p}(1+|D u|)^{q_{1}} \\
& =A^{\prime} \psi(x)^{\beta} u^{p}(1+|D u|)^{q}\left\{-1+(1+|D u|)^{-\left(q-q_{1}\right)}\right\} \\
& \left.\leq 0 \quad \text { (because } q \geq 1-p>q_{1}\right) .
\end{aligned}
$$

Hence $u(x)$ is an upper solution of the problem (4.1) with $h(x, u, \xi)$ given by (4.4). Now, since $\beta+2 \leq q_{1}<1-p$, by following the argument we have made under the condition $(i)$ we are reduced to absurdity. Therefore, the problem (1.1) still can not have a classical solution under the condition (ii). The proof of Theorem 4 is finished. Q. E. D.

\section{The Proofs of Theorem 5 and 6}

The nonexistence conclusion of Theorem 5 is a corollary of Theorem 4. The existence conclusions are corollaries of Theorem 1 and 3 . The uniqueness conclusion is a corollary of Lemma 4. Besides, from the proofs of Theorem 1 and 3 one sees directly that (2.13), (2.15) and the first inequality in (2.14) are valid. Thus to complete the proof of Theorem 5 the remaining thing is to prove (2.12) and the second inequality in (2.14).

Proof of (2.12). Let

$$
\begin{gathered}
\nu=(2+\beta-q) /(1-p-q), \\
K_{1}=\min _{\bar{\Omega}}\left(\lambda_{1} \psi^{2}+(1-\nu)|D \psi|^{2}\right), \quad K_{2}=\max _{\bar{\Omega}}\left(\lambda_{1} \psi^{2}+(1-\nu)|D \psi|^{2}\right), \\
K_{3}=\min _{\bar{\Omega}}\left(\psi^{2(1-\nu)}+\nu^{2}|D \psi|^{2}\right), \quad K_{4}=\max _{\bar{\Omega}}\left(\psi^{2(1-\nu)}+\nu^{2}|D \psi|^{2}\right),
\end{gathered}
$$


where $\lambda_{1}$ is the smallest eigenvalue of the operator $-\triangle: H_{0}^{1}(\Omega) \rightarrow H^{-1}(\Omega$. Since $0<\nu<1$ by virtue of the hypothesis, we have $K_{1}>0, K_{2}<\infty, K_{3}>0$ and $K_{4}<\infty$. Besides, we have

$$
\beta+\nu p+(\nu-1) q=(\beta+2 p+q) /(1-p-q)=\nu-2 .
$$

Now take a number $\varepsilon \in(0,1)$ sufficiently small such that

$$
\left\{\begin{array}{l}
C_{1}^{\prime} K_{4}^{q / 2}-\nu K_{2} \varepsilon^{1-p} \geq 0, \quad \text { if } q<0, \\
C_{1}^{\prime} K_{3}^{q / 2}-\nu K_{2} \varepsilon^{1-p-q} \geq 0, \quad \text { if } q \geq 0 ;
\end{array}\right.
$$

and take another number $E \geq 1$ sufficiently large such that

$$
\left\{\begin{array}{l}
C_{2}^{\prime} K_{3}^{q / 2}-\nu K_{1} E^{1-p} \leq 0, \quad \text { if } q<0, \\
C_{2}^{\prime} K_{4}^{q / 2}-\nu K_{1} E^{1-p-q} \leq 0, \quad \text { if } q \geq 0 .
\end{array}\right.
$$

In the above $C_{1}^{\prime}$ and $C_{2}^{\prime}$ are the positive constants such that

$$
C_{1}^{\prime} \psi(x)^{\beta} \leq a(x) \leq C_{2}^{\prime} \psi(x)^{\beta}, \quad \forall x \in \Omega,
$$

which is guaranteed by (1.7) and (2.2). With $\nu, \varepsilon$ and $E$ taken in this way, we assert that $\underline{u}(x)=\varepsilon \psi(x)^{\nu}$ and $\bar{u}(x)=E \psi(x)^{\nu}$ are a pair of lower and upper solutions of the problem (1.6). In fact, by (5.3) we have

$$
\begin{aligned}
& \triangle \underline{u}+a(x) \underline{u}^{p}\left(1+|D \underline{u}|^{2}\right)^{q / 2} \\
& \geq-\lambda_{1} \nu \varepsilon \psi^{\nu}-\nu(1-\nu) \varepsilon \psi^{\nu-2}|D \psi|^{2}+C_{1}^{\prime} \varepsilon^{p} \psi^{\beta+\nu p}\left(1+\varepsilon^{2} \nu^{2} \psi^{2(\nu-1)}|D \psi|^{2}\right)^{q / 2} \\
& \begin{array}{c}
\geq\left\{\begin{aligned}
-\nu \varepsilon \psi^{\nu-2}\left(\lambda_{1} \psi^{2}+(1-\nu)|D \psi|^{2}\right)+ & C_{1}^{\prime} \varepsilon^{p} \psi^{\beta+\nu p+(\nu-1) q}\left(\psi^{2(1-\nu)}+\nu^{2}|D \psi|^{2}\right)^{q / 2}, \\
& \text { if } q<0, \\
-\nu \varepsilon \psi^{\nu-2}\left(\lambda_{1} \psi^{2}+(1-\nu)|D \psi|^{2}\right)+ & C_{1}^{\prime} \varepsilon^{p+q} \psi^{\beta+\nu p+(\nu-1) q}\left(\psi^{2(1-\nu)}+\nu^{2}|D \psi|^{2}\right)^{q / 2}, \\
& \text { if } q \geq 0
\end{aligned}\right. \\
\geq\left\{\begin{array}{rr}
\varepsilon^{p} \psi^{\nu-2}\left\{C_{1}^{\prime}\left(\psi^{2(1-\nu)}+\nu^{2}|D \psi|^{2}\right)^{q / 2}-\nu \varepsilon^{1-p}\left(\lambda_{1} \psi^{2}+(1-\nu)|D \psi|^{2}\right)\right\}, \\
\text { if } q<0, \\
\varepsilon^{p+q} \psi^{\nu-2}\left\{C_{1}^{\prime}\left(\psi^{2(1-\nu)}+\nu^{2}|D \psi|^{2}\right)^{q / 2}-\nu \varepsilon^{1-p-q}\left(\lambda_{1} \psi^{2}+(1-\nu)|D \psi|^{2}\right)\right\}, \\
\text { if } q \geq 0
\end{array}\right. \\
\geq\left\{\begin{array}{rr}
\varepsilon^{p} \psi^{\nu-2}\left\{C_{1}^{\prime} K_{4}^{q / 2}-\nu K_{2} \varepsilon^{1-p}\right\}, & \text { if } q<0, \\
\varepsilon^{p+q} \psi^{\nu-2}\left\{C_{1}^{\prime} K_{3}^{q / 2}-\nu K_{2} \varepsilon^{1-p-q}\right\}, & \text { if } q \geq 0
\end{array}\right. \\
\geq 0 .
\end{array}
\end{aligned}
$$

Hence $\underline{u}(x)=\varepsilon \psi(x)^{\nu}$ is a lower solution of the problem (1.6). In a similar way we can prove that $\bar{u}(x)=E \psi(x)^{\nu}$ is an upper solution of the problem (1.6). This proves our assertion. Consequently, by comparison (Lemma 4 ) we get (2.12). Q. E. D.

The second inequality in (2.14) can be proved similarly. Namely, we can prove that for sufficiently large $E$, the function $\bar{u}(x)=E \psi(x)$ is an upper solution of the problem (1.6). Since the proof is simple, we omit it here. 
In order to prove Theorem 6 , we need the following two prelimilary lemmas:

Lemma 5 Let $\Omega$ be an bounded open set in $R^{n}$. Let $d(x)=d(x, \partial \Omega)$. Suppose that $u \in W^{2, r}(\Omega)$ for some $r \in(1, \infty)$. Then it follows that

$$
\|d(x) D u\|_{L^{r}(\Omega)} \leq C\left(\left\|d(x)^{2} \triangle u\right\|_{L^{r}(\Omega)}+\|u\|_{L^{r}(\Omega)}\right)^{\frac{1}{2}}\|u\|_{L^{r}(\Omega)}^{\frac{1}{2}},
$$

where $C$ is a constant independent of $\Omega$ and $u$.

This lemma is a corollary of $[25$, Theorem 17.1.3, p.6]. From this lemma we see that for any $\varepsilon>0$, there exists a corresponding constant $C(\varepsilon)$ independent of $\Omega$ and $u$ such that

$$
\|d(x) D u\|_{L^{r}(\Omega)} \leq \varepsilon\left\|d(x)^{2} \triangle u\right\|_{L^{r}(\Omega)}+C(\varepsilon)\|u\|_{L^{r}(\Omega)} .
$$

Lemma 6 Let $\Omega$ be a as before. Let $u \in W_{0}^{1, r}(\Omega)$ and $\triangle u \in L^{r}(\Omega)$ for some $r \in(1, \infty)$. Then $u \in W^{2, r}(\Omega)$ and the following inequality is valid:

$$
\|u\|_{W^{2, r}(\Omega)} \leq C\left(\|\triangle u\|_{L^{r}(\Omega)}+\|u\|_{L^{r}(\Omega)}\right),
$$

where $C$ is a constant independent of $u$.

This lemma is a corollary of the well-known Agman-Douglis-Nirenberg Theorem (c.f.[26] for instance).

We now establish two lemmas which give us more information about the boundary regularity of solutions of (1.6) than Theorem 6 . In fact, Theorem 6 is actually a corollary of the second lemma. In the following we always briefly write $d(x, \partial \Omega)$ as $d(x)$

Lemma 7 Suppose that $a(x)$ is locally Hölder continuous in $\Omega$. Suppose that $p<$ $1, q<1$ and either $q<1-p, \beta>\max \left(q-2, \frac{p q}{1-q}-2\right)$ or $q \geq 1-p, \beta>-p-1$. Let $u(x)$ be the solution of (1.6) obtained by Theorem 5 . Then we have

$$
\begin{gathered}
\int_{\Omega}\left|d(x)^{2} \Delta u\right|^{r} \mathrm{~d} x<\infty, \quad \forall r \in[1, \infty) \\
\int_{\Omega}|d(x) D u|^{r} \mathrm{~d} x<\infty, \quad \forall r \in[1, \infty) .
\end{gathered}
$$

Proof. We only give the proof under the assumption $q<1-p$. When $q \geq 1-p$ the proof is similar and is left to the reader. Let us make discussion according to different situations of the range of $p, q$ and $\beta$ differently.

Situation 1: $q \leq 0, \frac{p q}{1-q}-2<\beta<-p-1$. Let for sufficiently large $k, \Omega_{k}=\{x: x \in$ $\Omega, d(x)>1 / k\}$. Let $d_{k}(x)=d\left(x, \partial \Omega_{k}\right)\left(x \in \Omega_{k}\right)$. Then by (1.6), (1.7) and (2.12) we have

$$
\int_{\Omega_{k}}\left|d_{k}(x)^{2} \triangle u\right|^{r} \mathrm{~d} x \leq \int_{\Omega_{k}} d_{k}(x)^{2 r} a(x)^{r} u^{p r} \mathrm{~d} x \leq C \int_{\Omega} d(x)^{(2+\beta+\nu p) r} \mathrm{~d} x,
$$

where $\nu=(2+\beta-q) /(1-p-q)$. As one may easily verify, the assumption $\beta>\frac{p q}{1-q}-2$ implies that $2+\beta+\nu p>0$. Therefore, the last integral in (5.6) is finite. Now let $k \rightarrow \infty$. Then we obtain (5.4). To get (5.5) we apply Lemma 5 and (5.6) and obtain

$$
\int_{\Omega_{k}}\left|d_{k}(x) D u\right|^{r} \mathrm{~d} x \leq C \int_{\Omega} d(x)^{(2+\beta+\nu p) r} \mathrm{~d} x+C .
$$


Thus by letting $k \rightarrow \infty$ again we get (5.5).

Situation 2: $0<q<1, q-2<\beta<-p-1$. In this situation we have

$$
\int_{\Omega_{k}}\left|d_{k}(x)^{2} \triangle u\right|^{r} \mathrm{~d} x \leq C \int_{\Omega} d(x)^{(2+\beta+\nu p) r} \mathrm{~d} x+C \int_{\Omega_{k}} d(x)^{(2+\beta+\nu p-q) r}\left|d_{k}(x) D u\right|^{q r} \mathrm{~d} x
$$

where $\nu$ is as above. Since $2+\beta+\nu p-q=\frac{(2+\beta-q)(1-q)}{1-p-q}>0$, we get

$$
\int_{\Omega_{k}}\left|d_{k}(x)^{2} \triangle u\right|^{r} \mathrm{~d} x \leq C+C \int_{\Omega_{k}}\left|d_{k}(x) D u\right|^{q r} \mathrm{~d} x \leq C+C\left(\int_{\Omega_{k}}\left|d_{k}(x) D u\right|^{r} \mathrm{~d} x\right)^{q},
$$

where $C$ represents constant independent of $k$ and $u$. Now we first apply Lemma 5 and then apply Young's inequality. Then we get

$$
\int_{\Omega_{k}}\left|d_{k}(x)^{2} \triangle u\right|^{r} \mathrm{~d} x \leq C .
$$

As we have seen, (5.4) follows from a limitation process and (5.5) follows from an application of Lemma 5 and a similar limitation process.

Situation 3: $q \leq 0,-p-1 \leq \beta<1-p$. In this situation by (2.13) we have

$$
\int_{\Omega_{k}}\left|d_{k}(x)^{2} \triangle u\right|^{r} \mathrm{~d} x \leq C_{\nu_{1}} \int_{\Omega} d(x)^{\left(2+\beta+\nu_{1} p\right) r} \mathrm{~d} x
$$

where $\nu_{1}=\nu \in(0,1)$ for $p>0$ and 1 for $p \leq 0$, and $C_{\nu_{1}}$ represents constant depending on the choice of $\nu$. Since $\beta \geq-p-1$, we have $2+\beta+\nu_{1} p>0$ if $\nu$ is taken to be sufficiently near to 1 . Consequently, the integral on the right hand side of (5.8) is finite. Now by repeating the argument in the first situation we obtain (5.4) and (5.5).

Situation 4: $0<q<1,-p-1 \leq \beta<1-p$. In this situation we have

$$
\begin{aligned}
\int_{\Omega_{k}}\left|d_{k}(x)^{2} \Delta u\right|^{r} \mathrm{~d} x & \leq C \int_{\Omega} d(x)^{\left(2+\beta+\nu_{1} p\right) r} \mathrm{~d} x+C \int_{\Omega_{k}} d(x)^{\left(2+\beta+\nu_{1} p-q\right) r}\left|d_{k}(x) D u\right|^{q r} \mathrm{~d} x \\
& \leq C+C_{\nu_{1}}\left(\int_{\Omega_{k}} d(x)^{\frac{\left(2+\beta+\nu_{1} p-q\right) r}{1-q}} \mathrm{~d} x\right)^{1-q}\left(\int_{\Omega_{k}}\left|d_{k}(x) D u\right|^{r} \mathrm{~d} x\right)^{q},
\end{aligned}
$$

where $\nu_{1}$ is as before. Since $\beta \geq-p-1$ and $1-q>0$, we have $2+\beta+\nu_{1} p-q>0$ if $\nu$ for $p>0$ is taken sufficiently near to 1 . Hence by repeating the argument in situation 2 we again get (5.4) and (5.5).

Situation 5: $\beta \geq 1-p$. The proof is similar. We thus omit it here. Q. E. D.

Lemma 8 Suppose that $a(x)$ is locally Hölder continuous in $\Omega$. Suppose that $n \geq$ $2, p<1, q<1$ and either $q<1-p, \beta>\max \left(q-2, \frac{p q}{1-q}-2\right)$ or $q \geq 1-p, \beta>-p-1$. Let $u(x)$ be the solution of (1.6) obtained by Theorem 5 . Then there exists $r_{0} \in\left(\frac{n}{2}, \infty\right]$ such that

$$
\int_{\Omega}|\triangle u|^{r} \mathrm{~d} x<\infty, \quad \forall r \in\left[1, r_{0}\right) .
$$

Moreover, $r_{0}>n$ if $\beta>-p-1$.

Proof. Again we only give the proof under the assumption $q<1-p$. We make discussion according to different cases of the range of $p, q$ and $\beta$ differently. 
Case 1: $q \leq 0, \frac{p q}{1-q}-2<\beta \leq-p-1$. In this case we denote $r_{0}=-n(1-p-q) /\{\beta(1-$ $q)+p(2-q)\}$. It is not difficult to verify that $n / 2<r_{0} \leq n$. By making application of (1.6), (1.7) and (2.12) we obtain

$$
\int_{\Omega}|\triangle u|^{r} \mathrm{~d} x \leq C \int_{\Omega} d(x)^{(\beta+\nu p) r} \mathrm{~d} x, \forall r \in\left(1, r_{0}\right),
$$

where $\nu=(2+\beta-q) /(1-p-q)$. As one may easily verify, $r<r_{0}$ implies that $(\beta+\nu p) r>-n$. Therefore, the integral on the right hand side of the above inequality is finite. This proves (5.10).

Case 2: $0<q<1, q-2<\beta \leq-p-1$. In this case we denote $r_{0}=n(1-p-q) /\{2(1-$ $p-q)-(2+\beta-q)(1-q)\}$. It is evident that $n / 2<r_{0} \leq n$. Again by (1.6), (1.7) and (2.12) we have

$$
\int_{\Omega}|\triangle u|^{r} \mathrm{~d} x \leq C \int_{\Omega} d(x)^{(\beta+\nu p) r} \mathrm{~d} x+C \int_{\Omega} d(x)^{(\beta+\nu p-q) r}|d(x) D u|^{q r} \mathrm{~d} x, \quad \forall r \in\left(1, r_{0}\right),
$$

where $\nu$ is as in Case 1 . As one may easily verify, $r<r_{0}$ implies that $(\beta+\nu p-q) r>-n$. Now we choose a number $s>1$ sufficiently near to 1 such that $(\beta+\nu p-q) r s>-n$ and denote by $s^{\prime}$ the dual number of $s$. Then by applying Hölder inequality we get

$$
\int_{\Omega}|\triangle u|^{r} \mathrm{~d} x \leq C+C\left(\int_{\Omega}|d(x) D u|^{q r s^{\prime}} \mathrm{d} x\right)^{\frac{1}{s^{\prime}}}, \forall r \in\left(1, r_{0}\right) .
$$

Thus by virtue of Lemma 7 we obtain (5.10).

Case 3: $q \leq 0, \beta>-p-1$. In this case we denote $r_{0}=-n /(\beta+p)$ for $-p-1<\beta<-p$ and $r_{0}=\infty$ for $\beta \geq-p$. Since $\beta+p>-1$, we have $r_{0}>n$. Now for any $r \in\left[1, r_{0}\right)$ we choose for $p>0$ a number $\nu \in(0,1)$ sufficiently near to 1 such that $(\beta+\nu p) r>-n$. Then by $(1.6),(1.7),(2.13)$ and $(2.14)$ we have

$$
\int_{\Omega}|\Delta u|^{r} \mathrm{~d} x \leq C \int_{\Omega} d(x)^{\left(\beta+\nu_{1} p\right) r} \mathrm{~d} x<\infty .
$$

Hence (5.10).

Case 4: $0<q<\beta+p+1$. In this case we denote $r_{0}=-n /(\beta+p-q)$ for $-1<$ $\beta+p-q<0$ and $r_{0}=\infty$ for $\beta+p-q \geq 0$. Then $r_{0}>n$. For any $r \in\left[1, r_{0}\right)$ we choose for $p>0$ a number $\nu \in(0,1)$ sufficiently near to 1 such that $(\beta+\nu p-q) r>-n$. Then we have

$$
\begin{aligned}
\int_{\Omega}|\triangle u|^{r} \mathrm{~d} x & \leq C \int_{\Omega} d(x)^{\left(\beta+\nu_{1} p\right) r} \mathrm{~d} x+C \int_{\Omega} d(x)^{\left(\beta+\nu_{1} p\right) r}|D u|^{q r} \mathrm{~d} x \\
& \leq C+C\left(\int_{\Omega} d(x)^{\left(\beta+\nu_{1} p-q\right) r s} \mathrm{~d} x\right)^{\frac{1}{s}}\left(\int_{\Omega}|d(x) D u|^{q r s^{\prime}} \mathrm{d} x\right)^{\frac{1}{s^{\prime}}},
\end{aligned}
$$

where $s \in(1, \infty)$ is sufficiently near to 1 such that $\left(\beta+\nu_{1} p-q\right) r s>-n$ and $s^{\prime}$ is the dual number of $s$. By (5.4), we again get (5.10).

Case 5: $q>0$ and $q^{m} /\left(1+q+\cdots+q^{m-1}\right)<\beta+p+1 \leq q^{m-1} /\left(1+q+\cdots+q^{m-2}\right)$ $(m=2,3, \cdots)$. In this case we prove by induction that

$$
\begin{gathered}
\int_{\Omega}|\triangle u|^{r} \mathrm{~d} x<\infty, \text { if } 1 \leq r<n /\left\{q^{k}-\left(1+q+\cdots+q^{k-1}\right)(\beta+p+1)+1\right\} \\
k=1,2, \cdots, m .
\end{gathered}
$$


Indeed, from the deduction in Case 4 we see that (5.11) is valid for $k=1$. Suppose that (5.11) is valid for a $k \leq m-1$. Then by Lemma 6 and Sobolev's embedding theorem we get

$$
\int_{\Omega}|D u|^{r} \mathrm{~d} x<\infty, \text { if } 1 \leq r<n /\left\{q^{k}-\left(1+q+\cdots+q^{k-1}\right)(\beta+p+1)\right\} .
$$

Now let $1 \leq r<n /\left\{q^{k+1}-\left(1+q+\cdots+q^{k}\right)(\beta+p+1)+1\right\}$. Then

$$
\frac{\left\{q^{k}-\left(1+q+\cdots+q^{k-1}\right)(\beta+p+1)\right\} q r}{n}+\frac{-(\beta+p) r}{n}<1 .
$$

We have

$$
\beta+p+1 \leq \frac{q^{m-1}}{1+q+\cdots+q^{m-2}} \leq \frac{q^{k}}{1+q+\cdots+q^{k-1}} \leq \cdots \leq q<1 .
$$

Therefore, both terms in the left hand side of (5.13) are nonnegative, which implies that there exists a number $\lambda \in(0,1)$ such that

$$
1-\lambda>\frac{\left\{q^{k}-\left(1+q+\cdots+q^{k-1}\right)(\beta+p+1)\right\} q r}{n}, \quad \lambda>-\frac{(\beta+p) r}{n},
$$

or equivalently,

$$
\frac{q r}{1-\lambda}<\frac{n}{q^{k}-\left(1+q+\cdots+q^{k-1}\right)(\beta+p+1)}, \frac{(\beta+p) r}{\lambda}>-n .
$$

With $\lambda$ taken in this way, we choose for $p>0$ a number $\nu \in(0,1)$ sufficiently near to 1 such that $\frac{(\beta+\nu p) r}{n}>-n$. Then from (5.12) we get

$$
\begin{aligned}
\int_{\Omega}|\triangle u|^{r} \mathrm{~d} x & \leq C \int_{\Omega} d(x)^{\left(\beta+\nu_{1} p\right) r} \mathrm{~d} x+C \int_{\Omega} d(x)^{\left(\beta+\nu_{1} p\right) r}|D u|^{q r} \mathrm{~d} x \\
& \leq C+C\left(\int_{\Omega} d(x)^{\frac{\left(\beta+\nu_{1} p\right) r}{\lambda}} \mathrm{d} x\right)^{\lambda}\left(\int_{\Omega}|D u|^{\frac{q r}{1-\lambda}} \mathrm{d} x\right)^{1-\lambda}<\infty .
\end{aligned}
$$

Therefore, (5.11) holds for every $k=1,2, \cdots, m$.

Now we denote

$$
r_{0}=\frac{n}{q^{m}-\left(1+q+\cdots+q^{m-1}\right)(\beta+p+1)+1} .
$$

It is obvious that $r_{0}>n$ and (5.10) follows from (5.11) by taking $k=m$. The proof of (5.10) is finished. Q. E. D.

Proof of Theorem 6. The conclusions $(i)$ and $(i i)$ follow directly from Lemma 8, Lemma 6 and Sobolev-Morrey embedding theorem. We now give the proof of conclusion (iii). Denote

$$
f(x)=a(x) u(x)^{p}\left(1+|D u(x)|^{2}\right)^{\frac{q}{2}},
$$

and write

$$
f(x)=a(x) \tilde{d}(x)^{-\beta} \cdot \tilde{d}(x)^{\beta} u(x)^{p} \cdot\left(1+|D u(x)|^{2}\right)^{\frac{q}{2}} .
$$

Since $\beta+p>0$, by making application of $(2.13),(2.14),(2.15)$ and (2.16) we see that for $r>n$ sufficiently near to $n$,

$$
\int_{\Omega}\left|D\left(\tilde{d}(x)^{\beta} u(x)^{p}\right)\right|^{r} \mathrm{~d} x \leq C\left(\int_{\Omega}\left|\tilde{d}(x)^{\beta-1} u(x)^{p}\right|^{r} \mathrm{~d} x+\int_{\Omega}\left|\tilde{d}(x)^{\beta} u(x)^{p-1}\right|^{r} \mathrm{~d} x\right)<\infty .
$$

Therefore, $\tilde{d}(x)^{\beta} u(x)^{p} \in C^{\alpha}(\Omega)$ for some $\alpha \in(0,1)$. Thus from (5.14) we see that $f(x) \in$ $C^{\alpha}(\Omega)$ for some $\alpha \in(0,1)$. The conclusion (iii) then follows from the well-known Hölder's regularity theorem for elliptic boundary value problems (c.f.[26] for instance). Q. E. D. 


\section{References}

[1] Crandall M. G., Rabinowitz P. H. and Tartar L.. On the Dirichlet problem with singular nonlinearity, Comm. in P. D. E., 2(1977), 193-222.

[2] Taliaferro S. D.. A nonlinear singular boundary value problem, Nonlinear Anal., 3(1979), 897-904.

[3] Usami H.. On a singular elliptic boundary value problem in a ball, Nonlinear Anal., 13(1989), 1163-1170.

[4] Callegari A. and Nachman A.. Some singular nonlinear differential equations arising in boundary layer theory, J. Math. Anal. Appl., 64(1978), 96-105.

[5] Dunninger D. R. and Kurtz J. C.. Existence of solutions for some nonlinear singular boundary value problems, J. Math. Anal. Appl., 115(1986), 396-405.

[6] Gomes S. M.. On a singular nonlinear elliptic problem, SIAM J. Math. Anal., 17(1986), 1359-1369.

[7] Diaz J. L., Morel J. M. and Oswald L.. Elliptic equations with singular nonlinearity, Comm. in P. D. E.,12(1987), 1333-1344.

[8] Dalmasso R.. Solutions d'equations elliptiques semi-lineaires singulieres, Annali Mat. Pura Appl., 153(1988), 191-201.

[9] Bobisud L. E., O'Regan D. and Royalty W. D.. Solvability of some nonlinear boundary value problems, Nonlinear Anal., 12(1988), 855-869.

[10] Iscoe I.. On the supports of measure-valued critical branching Brownian motion, The Ann. of Probability, 16(1988), 200-211.

[11] Gatica J. A.. Singular nonlinear boundary value problems for second order ordinary differential equations, J. Diff. Equa., 79(1989), 62-78.

[12] Coclite M. M. and Palmieri G.. On a singular nonlinear Dirichlet problem, Comm. in P. D. E., 14(1989), 1315-1327.

[13] Senba T., Ebihara Y. and Furusho U.. Dirichlet problem for a semilinear elliptic equation with singular coefficients, Nonlinear Anal., 15(1990), 299-306.

[14] Choi Y. S.. A singular boundary value problem arising from near ignition analysis of falme structure, Diff. and Int. Equa., 4(1991), 891-895.

[15] Baxley J. V.. Some singular nonlinear boundary value problems, SIAM J. Math. Anal., $22(1991), 463-479$.

[16] Fink A. M., Gatica J. A., Hernandez G. E. and Waltman P.. Approximation of solutions of singular second order boundary value problems, SIAM J. Math. Anal,, 22(1991), 440-462.

[17] Lazer A. C. and Mckenna P. L.. On a singular nonlinear elliptic boundary value problem, Proc. Amer. Math. Soc., 111(1991), 721-730.

[18] Coclite M. M.. On a singular nonlinear Dirichlet problem III, Nonlinear Anal., 21(1993), $547-564$. 
[19] Cui Shangbin. Positive solutions for Dirichlet problems associated to semilinear elliptic equations with singular nonlinearity, Nonlinear Anal., 21(1993), 181-190.

[20] Cui Shangbin. Positive solutions for Dirichlet problems of singular semilinear elliptic equations, Sys. Sci. \& Math. Sci., 8(1995), 203-208.

[21] Zhang Z.. On a Dirichlet problem with a singular nonlinearity, J. Math. Anal. Appl., 194(1995), 103-113.

[22] Zhang Z.. Nonexistence of positive classical solutions of a singular nonlinear Dirichlet problem with a convection term, Nonlinear Anal., *(1995), *_*.

[23] Cui Shangbin. Some comparison and uniqueness theorems for nonlinear elliptic boundary value problems and nonlinear parabolic initial boundary value problems, Nonlinear Anal., 29(1997), 1079-1090.

[24] Noussair E. S.. On semilinear elliptic boundary value problems in unbounded domains, J. Diff. Equa., 41(1981), 334-348.

[25] Hörmander L.. The Analysis of Linear Partial Differential Operators, Vol. III, Berlin: Springer, 1985.

[26] Gilbarg D. and Trudinger N. S.. Elliptic Partial Differential Equations of Second Order, 2nd edition, Berlin: Springer, 1983. 